\title{
23. \\ Anwendung der elliptischen Funktionen auf ein Problem der Geometrie des Raumes.
}

(Von Herrn Clebsch.)

Hr. Cayley hat in den Philos. Transact. vom Jahre 1852 eine analytische Behandlung von Steiner's Ausdehnung des Malfattischen Problems gegeben, und hat dort die Aufgabe

Zu drei, auf einer Oberfläche zweiter Ordnung gegebenen, ebenen Kurven, drei andere zu finden, welche sich unter einander berühren, und deren jede zugleich zwei der gegebenen Kurven berührt, zurückgeführt auf ein System von Gleichungen, welches als specieller Fall in dem folgenden enthalten ist:

$$
\text { (1.) }\left\{\begin{array}{l}
\alpha+\beta(y+z)+\gamma y z=\delta \sqrt{1+y^{2} \cdot 1+z^{2}} \\
\alpha^{\prime}+\beta^{\prime}(z+x)+\gamma^{\prime} z x=\delta^{\prime} \sqrt{1+z^{2} \cdot 1+x^{2}} \\
\alpha^{\prime \prime}+\beta^{\prime \prime}(x+y)+\gamma^{\prime \prime} x y=\delta^{\prime \prime} \sqrt{1+x^{2} \cdot 1+y^{2}} .
\end{array}\right.
$$

Dies System ,von Gleichungen ist dort durch rein algebraische Betrachtungen gelöst, unter gewissen Bedingungen, welchen die Coefficienten genügen müssen; und so auch die algebraische Lösung der obigen Aufgabe vollständig gegeben.

Die Auflösung der Gleichungen (1.) läfst sich indefs auch aus einem andern Gesichtspunkte darstellen. Denn indem man jede der obigen Gleichungen mit der Form vergleicht, welche schon Euler für das Additionstheorem der elliptischen Funktionen gegeben hat, kann man die Gleichungen (1.) betrachten als die algebraischen Integrale von Gleichungen der Form

$$
\text { (2.) }\left\{\begin{array}{l}
\frac{d y}{\sqrt{Y}}+\frac{d z}{\sqrt{Z}}=0 \\
\frac{d z}{\sqrt{Z^{\prime}}}+\frac{d x}{\sqrt{X^{\prime}}}=0 \\
\frac{d x}{\sqrt{X^{\prime \prime}}}+\frac{d y}{\sqrt{Y^{\prime \prime}}}=0
\end{array}\right.
$$

wo $\boldsymbol{X}^{\prime}, \boldsymbol{X}^{\prime \prime}$ ganze Funktionen vierten Grades von $x$ sind, $\boldsymbol{Y}, \boldsymbol{Y}^{\prime \prime}$ von $y, \boldsymbol{Z}, \boldsymbol{Z}^{\prime}$ 
23. Clebsch, das Malfattische Problem auf den Oberflächen $2^{\text {ter }}$ Ordnung. 293

von $z$. Und indem man diese Funktionen wirklich ausrechnet, findet man für die Gleichungen (2.) die folgenden:

$$
\begin{gathered}
0=\frac{d y}{\sqrt{1+\bar{y}^{2}} \sqrt{\left(\delta^{2}-\beta^{2}-\alpha^{2}\right)-2 \gamma(\alpha+\beta) y+\left(\delta^{2}-\gamma^{2}-\beta^{2}\right) y^{2}}} \\
+\frac{d z}{\sqrt{1+z^{2}} \sqrt{\left(\delta^{2}-\beta^{2}-\alpha^{2}\right)-2 \gamma(\alpha+\beta) z+\left(\delta^{2}-\overline{\left.\gamma^{2}-\beta^{2}\right) z^{2}}\right.}} \\
\text { etc. }
\end{gathered}
$$

Und um die Integrale dieser Gleichungen vergleichen zu können, mufs man unter den Wurzelzeichen bis auf einen constanten Faktor immer dieselbe Funktion haben, d. h. die Ausdrücke

$$
\begin{gathered}
\left(\delta^{2}-\beta^{2}-\alpha^{2}\right)-2 \gamma(\alpha+\beta) u+\left(\delta^{2}-\gamma^{2}-\beta^{2}\right) u^{2} \\
\left(\delta^{\prime 2}-\beta^{\prime 2}-\alpha^{\prime 2}\right)-2 \gamma^{\prime}\left(\alpha^{\prime}+\beta^{\prime}\right) u+\left(\delta^{\prime 2}-\gamma^{\prime 2}-\beta^{\prime 2}\right) u^{2} \\
\left(\delta^{\prime \prime 2}-\beta^{\prime \prime 2}-\alpha^{\prime 2}\right)-2 \gamma^{\prime \prime}\left(\alpha^{\prime \prime}+\beta^{\prime \prime}\right) u+\left(\delta^{\prime \prime 2}-\gamma^{\prime \prime 2}-\beta^{\prime \prime 2}\right) u^{2}
\end{gathered}
$$

dürfen sich nur um constante Faktoren unterscheiden. Daher erhält man die Bedingungen

$$
\begin{aligned}
& \delta^{2}-\beta^{2}-\alpha^{2}: 2 \gamma(\alpha+\beta): \delta^{2}-\gamma^{2}-\beta^{2} \\
= & \delta^{\prime 2}-\beta^{\prime 2}-\alpha^{\prime 2}: 2 \gamma^{\prime}\left(\alpha^{\prime}+\beta^{\prime}\right): \delta^{\prime 2}-\gamma^{\prime 2}-\beta^{\prime 2} \\
= & \delta^{\prime \prime 2}-\beta^{\prime \prime 2}-\alpha^{\prime \prime 2}: 2 \gamma^{\prime \prime}\left(\alpha^{\prime \prime}+\beta^{\prime \prime}\right): \delta^{\prime \prime 2}-\gamma^{\prime \prime 2}-\beta^{\prime \prime 2}
\end{aligned}
$$

für die Anwendbarkeit dieser Methode; und es sind dies genau die von Hrn. Cayley aufgestellten Bedingungen.

Man wird durch diese Betrachtung darauf geführt, das Problem nicht auf die Gleichungen (1.) zurückzuführen, sondern auf andere, welche sich an die gewöhnliche Form des Additionstbeorems anschliefsen, wo man dann zugleich die Constanten, welche in die Integrale der Gleichungen (2.) eingehen, durch die Constanten des Problems bestimmt. Zu diesem Ende aber mufs ich die Behandlung der Aufgabe von Anfang an noch einmal kurz darstellen.

Für das Malfattische Problem selbst, wo man auf Kreisfunktionen geführt wird, hat schon Hr. Prof. Schellbach diesen Weg eingeschlagen und im 45sten Bande dieses Journals die sehr einfachen Resultate bekannt gemacht.

\$. 1.

Die Bedingung dafs drei auf einer Oberfläche zweiter Ordnung

$$
u=\sum_{i=1}^{i=4} \sum_{k=1}^{k=4} u_{i k} x_{i} x_{k}=0
$$

gegebene ebene Kurven einander berühren, erhält man leicht, indem man be- 
294 23. Clebsch, das Malfattische Problem auf den Oberflüchen $2^{\text {ter }}$ Ordnung.

merkt, dafs jede Tangentenebene der Oberfläche:

$$
\boldsymbol{P}=p_{1} x_{1}+p_{2} x_{2}+p_{3} x_{3}+p_{4} x_{4}=0
$$

der Gleichung genügen mufs

$$
\text { (3.) } \quad 0=\left|\begin{array}{lllll}
u_{11} & u_{12} & u_{13} & u_{14} & p_{1} \\
u_{21} & u_{22} & u_{23} & u_{24} & p_{2} \\
u_{31} & u_{32} & u_{33} & u_{34} & p_{3} \\
u_{41} & u_{42} & u_{43} & u_{44} & p_{4} \\
p_{1} & p_{2} & p_{3} & p_{4} & 0
\end{array}\right|
$$

Denn sind nun die Ebenen der gegebenen Kurven

$$
\text { (4.) }\left\{\begin{array}{l}
\boldsymbol{C}=c_{1} x_{1}+c_{2} x_{2}+c_{3} x_{3}+c_{4} x_{4}=0 \\
C^{\prime}=c_{1}^{\prime} x_{1}+c_{2}^{\prime} x_{2}+c_{3}^{\prime} x_{3}+c_{4}^{\prime} x_{4}=0 \\
C^{\prime \prime}=c_{1}^{\prime \prime} x_{1}+c_{2}^{\prime \prime} x_{2}+c_{3}^{\prime \prime} x_{3}+c_{4}^{\prime \prime} x_{4}=0
\end{array}\right.
$$

so müssen alle Tangentenebenen, welche durch den Schnittpunkt von $C, C^{\prime}, C^{\prime \prime}$ gehen, und welche also die Form haben

$$
\boldsymbol{P}=\lambda \boldsymbol{C}+\lambda^{\prime} \boldsymbol{C}^{\prime}+\lambda^{\prime \prime} \boldsymbol{C}^{\prime \prime}=\mathbf{0}
$$

einer Gleichung genügen

$$
0=q^{00} \lambda^{2}+q^{11} \lambda^{\prime 2}+q^{22} \lambda^{\prime \prime 2}+2 q^{01} \lambda \lambda^{\prime}+2 q^{12} \lambda^{\prime} \lambda^{\prime \prime}+2 q^{20} \lambda^{\prime \prime} \lambda,
$$

welche man erhält, indem in (3.)

$$
p_{i}=\lambda c_{i}+\lambda^{\prime} c_{i}^{\prime}+\lambda^{\prime \prime} c_{i}^{\prime \prime}
$$

gesetzt wird. Sollen nun die Schnittkurven der $C$ mit der Oberfläche einander berühren, so kann man durch die Schnittlinie zweier $\boldsymbol{C}$ nur immer eine Tangentenebene an die Oberfläche legen, d. h. nur je eine Ebene finden der Form

$$
\lambda C+\lambda^{\prime} C^{\prime}=0 \quad \lambda^{\prime} C^{\prime}+\lambda^{\prime \prime} C^{\prime \prime}=0 \quad \lambda^{\prime \prime} C^{\prime \prime}+\lambda C=0
$$

welche der Gleichung (5.) genügt. Also mufs diese Gleichung in ein vollständiges Quadrat übergehen, sobald ein $\lambda$ verschwindet, d. h. es mufs sein

$$
\text { (6.) } \quad\left\{\begin{array}{l}
q^{00} q^{11}=\left(q^{01}\right)^{2} \\
q^{11} q^{22}=\left(q^{12}\right)^{2} \\
q^{22} q^{00}=\left(q^{20}\right)^{2}
\end{array} \quad \text { wo } \quad q^{i k}=\left|\begin{array}{lllll}
u_{11} & u_{12} & u_{13} & u_{14} & c_{1}^{i} \\
u_{21} & u_{22} & u_{23} & u_{24} & c_{2}^{i} \\
u_{31} & u_{32} & u_{33} & u_{34} & c_{3}^{i} \\
u_{41} & u_{42} & u_{43} & u_{44} & c_{4}^{i} \\
c_{1}^{k} & c_{2}^{k} & c_{3}^{k} & c_{4}^{k} & 0
\end{array}\right| .\right.
$$


23. Clebsch, das Malfattische Problem auf den Oberfä̈chen $2^{\text {ter }}$ Ordnung. 295

Würde hiedurch die rechte Seite von Gleichung (5.) im Allgemeinen für die $\lambda$ ein vollständiges Quadral, so träte zn den Gleichungen (6.) noch diese hinzu:

$$
\begin{gathered}
q^{(0)} \cdot q^{11} \cdot q^{22}=q^{(11} \cdot q^{12} \cdot q^{20} \quad \text { oder } \\
\left|\begin{array}{lll}
q^{(1)} & q^{(11} & q^{(12} \\
q^{(11} & q^{11} & q^{12} \\
q^{(12} & q^{12} & q^{22}
\end{array}\right|=0
\end{gathered}
$$

Bezeichnet man nun durch $\Delta$ die Determinante von $u$, durch $\Delta_{i k}$ ihre Differentialquotienten, so hat man auch

$$
\text { (7.) } \quad q^{i \hbar}=\sum_{m=1}^{m=4} \sum_{n=1}^{n=4} \Delta_{m n} c_{m}^{i} c_{n}^{k}
$$

und dies in die letzte Gleichung eingesetzt, giebt derselben nach den bekannten Sätzen über die Multiplication der Determinanten, die Form

$$
\mathbf{0}=\Delta^{3} \cdot \sum_{m=1}^{m=4} \sum_{n=1}^{n=4} \boldsymbol{u}_{m n} \boldsymbol{C}_{t,} \boldsymbol{C}_{n}
$$

wo $C_{1}, C_{2}, C_{3}, C_{4}$ die Koordinaten des Schnittpunkts von $\boldsymbol{C}, C^{\prime}, C^{\prime \prime}$ bedeuten. Verschwände hier $A$, so wäre die Oberfläche ein Kegel; verschwände der zweite Faktor, so rückte dieser Schnittpunkt in die Oberfläche. Indem man beide Fälle ausschliefsı, kann man also den Gleichungen (6.) die Bestimmung hinzufügen

$$
\text { (8.) } q^{(0)} \cdot q^{11} \cdot q^{22}+q^{(11} \cdot q^{12} \cdot q^{20}=0 \text {. }
$$

\$. 2 .

Sind in unsrer Aufgabe nun die gegebenen Ebenen $A=0, A^{\prime}=0$, $\boldsymbol{A}^{\prime \prime}=0$, die gesuchten $\boldsymbol{B}=0, \boldsymbol{B}^{\prime}=0, B^{\prime \prime}=0$, und

$$
\text { (9.) }\left\{\begin{array}{l}
A^{i}=a_{1}^{i} x_{1}+a_{2}^{i} x_{2}+a_{3}^{i} x_{3}+a_{4}^{i} x_{4} \\
\boldsymbol{B}^{i}=b_{1}^{i} x_{1}+b_{2}^{i} x_{2}+b_{3}^{i} x_{3}+b_{4}^{i} x_{4},
\end{array}\right.
$$

so giebt es unter diesen Ebenen 4 Systeme, welche sich verhalten sollen wie die $C$ des vorigen Paragraphen, nämlich
1. $\boldsymbol{A} \boldsymbol{B}^{\prime} \boldsymbol{B}^{\prime \prime}$
2. $\boldsymbol{B}^{\prime} \boldsymbol{A}^{\prime} \boldsymbol{B}^{\prime \prime}$
3. $\boldsymbol{B} \boldsymbol{B}^{\prime} \boldsymbol{A}^{\prime \prime}$
4. $\boldsymbol{B} \boldsymbol{B}^{\prime} \boldsymbol{B}^{\prime \prime}$.

Für jedes dieser Systeme würden wir aus (6.) drei Gleichungen erbalten und aus (8.) eine Zeichenbestimmung. Man erhält so 12 Gleichungen, die sich indefs unmittelbar auf 9 reduziren und benutzt werden können, um die Verhältnisse der $b$ zu bestimmen. 
Um indefs absolute Bestimmungen zu erhalten, wollen wir die Gleichungen hinzufügen:

$$
\text { (10.) } \begin{cases}\Sigma \Sigma \Delta_{i k} a_{i}^{0} a_{k}^{0}=-1 & \sum \Sigma \Delta_{i k} b_{i}^{0} b_{k}^{\prime \prime}=-1 \\ \Sigma \Sigma A_{i k} a_{i}^{\prime} a_{k}^{\prime}=-1 & \sum \Sigma A_{i k} b_{i}^{\prime} b_{k}^{\prime}=-1 \\ \Sigma \Sigma \Delta_{i k} a_{i}^{\prime} a_{k}^{\prime \prime}=-1 & \sum \Sigma A_{i k} b_{i}^{\prime \prime} b_{k}^{\prime \prime}=-1,\end{cases}
$$

Annahmen, welche immer möglich sind, sobald nur keine der Ebenen $\boldsymbol{A}$ eine Berührungsebene der Oberfläche wird. So werden nunmehr alle $q$ mit gleichen oberen Indices $=--1$, und die Gleichungen $(6,8$.$) geben das Schema:$

wo die $\varepsilon \pm 1$ bedeuten.

$$
\text { (11.) }\left\{\begin{array}{l}
q^{12}=\varepsilon \\
q^{20}=\varepsilon_{1} \\
q^{101}=\varepsilon_{2}
\end{array} \quad \varepsilon_{1} \varepsilon_{2} \varepsilon=1\right.
$$

Stellt man unter dieser Form jetzt die Bedingungen der Aufgabe wirklich hin, so erhält man:

$$
\begin{cases}\Sigma \Sigma \Delta_{i k} b_{i} a_{k}^{\prime}=-\varepsilon_{0}^{\prime} & \Sigma \Sigma \Delta_{i k} b_{i}^{\prime} a_{k}=-\varepsilon_{1}^{\prime \prime} \\ \Sigma \Sigma \Delta_{i k} b_{i}^{\prime} a_{k}^{\prime \prime}=-\varepsilon_{1}^{\prime \prime} & \Sigma \Sigma \Delta_{i k} b_{i}^{\prime \prime} a_{k}^{\prime}=-\varepsilon_{2}^{\prime} \\ \Sigma \Sigma \Delta_{i k} b_{i}^{\prime \prime} a_{k}=-\varepsilon_{2}^{0} & \Sigma \Sigma \Delta_{i k} b_{i} a_{k}^{\prime \prime}=-\varepsilon_{0}^{\prime \prime} \\ \Sigma \Sigma \Delta_{i k} b_{i}^{\prime} b_{k}^{\prime \prime}=\varepsilon_{1}^{\prime} \varepsilon_{2}^{\prime \prime} & \\ \Sigma \Sigma \Delta_{i k} b_{i}^{\prime \prime} b_{k}=\varepsilon_{2}^{\prime} \varepsilon_{0}^{\prime} & \varepsilon_{1}^{\prime \prime} \varepsilon_{2}^{0} \varepsilon_{2}^{\prime} \varepsilon_{0}^{\prime} \varepsilon_{0}^{\prime \prime} \varepsilon_{1}^{\prime \prime}=1 \\ \Sigma \Sigma \Delta_{i k} b_{i} b_{k}^{\prime}=\varepsilon_{0}^{\prime \prime} \varepsilon_{1}^{\prime \prime} & \end{cases}
$$

wo wieder die $\varepsilon$ die positive oder negative Einheit bezeichnen. Durch diese Gleichungen und die Gleichungen (10.) sind die b nun vollständig bestimmt.

\section{S. 3.}

Man führt leicht diese zwölf Gleichungen auf drei zurück, indem man sich eines Satzes über die Determinanten erinnert, welchen Hr. Prof. Hesse im 49sten Bande dieses Journals p. 252 gegeben hat, und welcher uns in einem speziellen Falle das folgende Lemma giebt.

„Multiplicirt man die symmetrische Determinante

$$
\boldsymbol{P}=\left|\begin{array}{llll}
\Delta_{11} & \Delta_{12} & \Delta_{13} & \Delta_{14} \\
\Delta_{21} & \Delta_{22} & \Delta_{23} & \Delta_{24} \\
\Delta_{31} & \Delta_{32} & \Delta_{33} & \Delta_{34} \\
\Delta_{41} & \Delta_{42} & \Delta_{43} & \Delta_{44}
\end{array}\right|
$$


23. Clebsch, das Malfattische Problem auf den Oberflächen $\mathbf{2}^{\text {ter }}$ Ordnung. 297 „mit dem Quadral der Determinante:

$$
\boldsymbol{R}=\left|\begin{array}{llll}
a_{1}^{0} & a_{1}^{\prime} & a_{1}^{\prime \prime} & a_{1}^{\prime \prime \prime} \\
a_{2}^{0} & a_{2}^{\prime} & a_{2}^{\prime \prime} & a_{2}^{\prime \prime \prime} \\
a_{3}^{\prime \prime} & a_{3}^{\prime} & a_{3}^{\prime \prime} & a_{3}^{\prime \prime \prime} \\
a_{4}^{0} & a_{4}^{\prime} & a_{4}^{\prime \prime} & a_{4}^{\prime \prime \prime}
\end{array}\right|,
$$

"so entsteht wiederum eine symmetrische Determinante:

$$
\boldsymbol{Q}=\left|\begin{array}{llll}
\boldsymbol{D}_{(1)} & \boldsymbol{D}_{01} & \boldsymbol{D}_{02} & \boldsymbol{D}_{03} \\
\boldsymbol{D}_{10} & \boldsymbol{D}_{11} & \boldsymbol{D}_{12} & \boldsymbol{D}_{13} \\
\boldsymbol{D}_{20} & \boldsymbol{D}_{21} & \boldsymbol{D}_{22} & \boldsymbol{D}_{23} \\
\boldsymbol{D}_{30} & \boldsymbol{D}_{31} & \boldsymbol{D}_{32} & \boldsymbol{D}_{33}
\end{array}\right|
$$

"wo

$$
\text { (13.) } \boldsymbol{D}_{p q}=\sum_{i=1}^{i=4} \sum_{k=1}^{k=4} \Delta_{i k} a_{i}^{p} a_{k}^{q} . "
$$

Lassen wir nun den $\Delta$ und $a$ die Bedeutungen, welche ihnen im Vorigen beigelegt waren, und setzen nur

$$
\text { (14.) } \quad a_{i}^{\prime \prime \prime}=\lambda b_{i}+\lambda^{\prime} b_{i}^{\prime}+\lambda^{\prime \prime} b_{i}^{\prime \prime},
$$

so geht das Produkt $\boldsymbol{P} . \boldsymbol{R}^{2}$ in einen Ausdruck über, welcher in den $\lambda$ sich als das Quadrat eines lineären darstellt. Dasselbe also mufs geschehen mit der Determinante $\boldsymbol{Q}$; und ist dieselbe, nach den $\lambda$ geordnet:

$$
\boldsymbol{Q}=v_{00} \lambda^{2}+v_{11} \lambda^{\prime 2}+v_{22} \lambda^{\prime \prime 2}+2 v_{01} \lambda \lambda^{\prime}+2 v_{12} \lambda^{\prime} \lambda^{\prime \prime}+2 v_{20} \lambda^{\prime \prime} \lambda,
$$

so müssen die $v$ den Gleichungen genügen:

$$
\left\{\begin{array}{l}
v_{(0)} v_{11}=v_{01}^{2} \\
v_{11} v_{22}=v_{12}^{2} \\
v_{22} v_{00}=v_{20}^{2}
\end{array} \quad v_{(0)} v_{11} v_{22}=v_{01} v_{12} v_{20}\right.
$$

Inzwischen werden in Folge der Gleichungen (10.), (13.) $\boldsymbol{D}_{00}, \boldsymbol{D}_{11}, \boldsymbol{D}_{22}$ sămmtlich $=-1$; und

$$
\boldsymbol{D}_{33}=-\left[\lambda^{2}+\lambda^{\prime 2}+\lambda^{\prime \prime 2}-2 \varepsilon_{2}^{\prime \prime} \varepsilon_{1}^{0} \lambda^{\prime} \lambda^{\prime \prime}-2 \varepsilon_{0}^{\prime} \varepsilon_{2}^{\prime} \lambda^{\prime \prime} \lambda-2 \varepsilon_{1}^{\prime \prime} \varepsilon_{0}^{\prime \prime} \lambda \lambda^{\prime}\right]
$$

Setzen wir ferner

$$
\text { (17.) } \begin{cases}-\alpha=\Sigma \Sigma \Delta_{i k} a_{i}^{\prime} a_{k}^{\prime \prime} & -\varepsilon_{0}^{0}\left(1+y_{0}\right)=\Sigma \Sigma \Delta_{i k} a_{i} b_{k} \\ -\alpha_{1}=\sum \Sigma \Delta_{i k} a_{i}^{\prime \prime} a_{k} & -\varepsilon_{1}^{\prime}\left(1+y_{1}\right)=\Sigma \Sigma \Delta_{i k} a_{i}^{\prime} b_{k}^{\prime} \\ -\alpha_{2}=\sum \Sigma \Delta_{i k} a_{i} a_{k}^{\prime} & -\varepsilon_{2}^{\prime \prime}\left(1+y_{2}\right)=\Sigma \Sigma \Delta_{i k} a_{i}^{\prime \prime} b_{k}^{\prime \prime}\end{cases}
$$

wo die $\varepsilon_{i}^{i}$ wieder \pm 1 sein sollen und später ihre nähere Bestimmung finden 38 * 
mögen; so wird

$$
\begin{array}{ll}
\boldsymbol{D}_{12}=-\alpha & \boldsymbol{D}_{03}=-\left\{\varepsilon_{0}^{0} \lambda\left(1+y_{0}\right)+\varepsilon_{1}^{0} \lambda^{\prime}+\varepsilon_{2}^{0} \lambda^{\prime \prime}\right\} \\
\boldsymbol{D}_{20}=-\alpha_{1} & \boldsymbol{D}_{13}=-\left\{\varepsilon_{1}^{\prime} \lambda+\varepsilon_{1}^{\prime} \lambda^{\prime}\left(1+y_{1}\right)+\varepsilon_{2}^{\prime} \lambda^{\prime \prime}\right\} \\
\boldsymbol{D}_{01}=-\alpha_{2} & \boldsymbol{D}_{23}=-\left\{\varepsilon_{0}^{\prime \prime} \lambda+\varepsilon_{1}^{\prime \prime} \lambda^{\prime \prime}+\varepsilon_{2}^{\prime \prime} \lambda^{\prime \prime}\left(1+y_{2}\right)\right\} .
\end{array}
$$

Die Gleichungen (16.) enthalten also nun allein die $y$ als unbekannte Gröfsen, da in den $\boldsymbol{D}$ andere nicht vorkommen, und genügen also zur Bestimmung derselben.

Bilden wir nun die Determinante $\boldsymbol{Q}$, so können wir offenbar zunächst die negativen Zeichen sämmtlich auslassen. Ferner setze ich an die Stelle der $\lambda$ die Gröfsen

$$
\mu=\varepsilon_{1}^{\prime \prime} \varepsilon_{2}^{\prime \prime} \lambda \quad \mu^{\prime}=\varepsilon_{2}^{\prime} \varepsilon_{0}^{\prime} \lambda^{\prime} \quad \mu^{\prime \prime}=\varepsilon_{0}^{\prime \prime} \varepsilon_{1}^{\prime \prime} \lambda^{\prime \prime} ;
$$

alsdann gehen die $\boldsymbol{D}_{03}, \boldsymbol{D}_{13}, \boldsymbol{D}_{23}, \boldsymbol{D}_{33}$ über in:

$$
\begin{aligned}
D_{33}= & -\left(\mu^{2}+\mu^{\prime 2}+\mu^{\prime \prime 2}\right) \\
& +2\left(\mu \mu^{\prime}+\mu^{\prime} \mu^{\prime \prime}+\mu^{\prime \prime} \mu\right)
\end{aligned}
$$

$$
\begin{aligned}
& \boldsymbol{D}_{03}=-\varepsilon_{1}^{\prime \prime} \varepsilon_{2}^{\prime} \varepsilon_{1}^{\prime}\left\{-\mu\left(1+y_{0}\right)+\mu^{\prime}+\mu^{\prime \prime}\right\} \\
& \boldsymbol{D}_{13}=-\varepsilon_{2}^{\prime} \varepsilon_{0}^{\prime \prime} \varepsilon_{1}^{\prime \prime}\left\{\mu-\mu^{\prime}\left(1+y_{1}\right)+\mu^{\prime \prime}\right\} \\
& \boldsymbol{D}_{23}=-\varepsilon_{0}^{\prime \prime} \varepsilon_{1}^{0} \varepsilon_{2}^{\prime \prime}\left\{\mu+\mu^{\prime}-\mu^{\prime \prime}\left(1+y_{2}\right)\right\}
\end{aligned}
$$

sobald man setzt

$$
\text { (18.) }-\varepsilon_{0}^{0}=\varepsilon_{2}^{\prime} \varepsilon_{0}^{\prime} \varepsilon_{2}^{\prime \prime}
$$$$
-\varepsilon_{1}^{\prime}=\varepsilon_{0}^{\prime \prime} \varepsilon_{1}^{\prime \prime} \varepsilon_{0}^{\prime}
$$$$
-\varepsilon_{2}^{\prime \prime}=\varepsilon_{1}^{0} \varepsilon_{2}^{0} \varepsilon_{1}^{\prime \prime} .
$$

Endlich noch kann man bei der Bildung der Determinante die Horizontalreihen und Vertikalreihea resp. mit $\varepsilon_{1}^{\prime \prime} \varepsilon_{2}^{\prime} \varepsilon_{0}^{\prime}, \varepsilon_{2}^{\prime} \varepsilon_{0}^{\prime \prime} \varepsilon_{1}^{\prime \prime}, \varepsilon_{0}^{\prime \prime} \varepsilon_{1}^{\prime \prime} \varepsilon_{2}^{\prime \prime}$ multiplicirt denken. Alsdann erhält man

$$
\left|\begin{array}{cccc|}
1 & \varepsilon_{2}^{0} \varepsilon_{2}^{\prime} \alpha_{2} & \varepsilon_{1}^{\prime \prime} \varepsilon_{1}^{0} \alpha_{1} & -\mu\left(1+y_{0}\right)+\mu^{\prime}+\mu^{\prime \prime} \\
\varepsilon_{2}^{0} \varepsilon_{2}^{\prime} \alpha_{2} & 1 & \varepsilon_{0}^{\prime} \varepsilon_{0}^{\prime \prime} \alpha & \mu-\mu^{\prime}\left(1+y_{1}\right)+\mu^{\prime \prime} \\
\varepsilon_{1}^{\prime \prime} \varepsilon_{1}^{0} \alpha_{1} & \varepsilon_{0}^{\prime} \varepsilon_{0}^{\prime \prime} \alpha & 1 & \mu+\mu^{\prime}-\mu^{\prime \prime}\left(1+y_{2}\right) \\
-\mu\left(1+y_{0}\right)+\mu^{\prime}+\mu^{\prime \prime}, \mu-\mu^{\prime}\left(1+y_{1}\right)+\mu^{\prime \prime}, \mu+\mu^{\prime}-\mu^{\prime \prime}\left(1+y_{2}\right), \mu^{2}+\mu^{\prime 2}+\mu^{\prime 2}-2 \mu \mu^{\prime}-2 \mu \mu^{\prime \prime}-2 \mu^{\prime} \mu^{\prime \prime}
\end{array}\right|
$$

Dies lehrt zunächst, dafs man aus einer Lösung der Aufgabe, für welche etwa sämmtliche $\varepsilon=+1$ sein mögen, vier erhält, indem man an die Stelle von $\alpha, \alpha_{1}, \alpha_{2}$ die Gröfsen $\varepsilon_{0}^{\prime} \varepsilon_{0}^{\prime \prime} \alpha$ etc. setzt, d. h. statt der $\alpha$ dieselben Gröfsen mit verändertem Vorzeichen, doch so dafs das Produkt derselben ungeändert bleibt. Ich nehme daher der Einfachheit wegen die $\varepsilon=1$ an, oder lasse sie in die $\alpha$ eingehen.

Wir müssen nun die $v$ genauer untersuchen, und zwar können wir sie jetzt die Coefficienten der $\mu$ bedeuten lassen. Es ist 
23. Clebsch, das Malfattische Problem auf den Oberfü̈chen $2^{\text {ter }}$ Ordnung. 299

$$
\begin{aligned}
& v_{10}=\left|\begin{array}{cccc}
1 & \alpha_{2} & \alpha_{1} & -\left(1+y_{0}\right) \\
\alpha_{2} & 1 & \alpha & 1 \\
\alpha_{1} & \alpha & 1 & 1 \\
-\left(1+y_{0}\right) & 1 & 1 & 1
\end{array}\right| \\
& =\left|\begin{array}{cccc}
1 & \alpha_{2} & \alpha_{1} & -1 \\
\alpha_{2} & 1 & \alpha & 1 \\
\alpha_{1} & \alpha & 1 & 1 \\
-1 & 1 & 1 & 1
\end{array}\right|+2 y_{0}\left|\begin{array}{ccc}
\alpha_{2} & 1 & \alpha \\
\alpha_{1} & \alpha & 1 \\
-1 & 1 & 1
\end{array}\right|+y_{0}^{2}\left(\alpha^{2}-1\right) \\
& v_{12}=\left|\begin{array}{cccc}
1 & \alpha_{2} & \alpha_{1} & 1 \\
\alpha_{2} & 1 & \alpha & 1 \\
\alpha_{1} & \alpha & 1 & -\left(1+y_{2}\right) \\
1 & -\left(1+y_{1}\right) & 1 & -1
\end{array}\right| \\
& =\left|\begin{array}{cccc}
1 & \alpha_{2} & \alpha_{1} & 1 \\
\alpha_{2} & 1 & \alpha & 1 \\
\alpha_{1} & \alpha & 1 & -1 \\
1 & -1 & 1 & -1
\end{array}\right|-y_{1}\left|\begin{array}{ccc}
1 & \alpha_{1} & 1 \\
\alpha_{2} & \alpha & 1 \\
\alpha_{1} & 1 & -1
\end{array}\right|-y_{2}\left|\begin{array}{ccc}
1 & \alpha_{2} & 1 \\
\alpha_{1} & \alpha & 1 \\
\alpha_{2} & 1 & -1
\end{array}\right|+y_{1} y_{2}\left(\alpha-\alpha_{1} \alpha_{2}\right)
\end{aligned}
$$

Wendet man in diesen Determinanten das gewöhnliche Mittel an sie zu vereinfachen, indem man die letzte Horizontalreihe zu den andern addirt oder abzieht, so erhält man:

(20.)

$$
\begin{aligned}
\left\{\begin{aligned}
v_{10} & =\left|\begin{array}{ccc}
0 & \alpha_{2}+1 & \alpha_{1}+1 \\
\alpha_{2}+1 & 0 & \alpha-1 \\
\alpha_{1}+1 & \alpha-1 & 0
\end{array}\right|+2 y_{0}\left|\begin{array}{cc}
\alpha_{2}+\alpha & 1-\alpha \\
\alpha_{1}+1 & \alpha-1
\end{array}\right|+y_{0}^{2}\left(\alpha^{2}-1\right) \\
& =(\alpha-1)\left\{2\left(\alpha_{1}+1\right)\left(\alpha_{2}+1\right)+2 y_{0}\left(\alpha+\alpha_{1}+\alpha_{2}+1\right)+y_{0}^{2}(\alpha+1)\right\} \\
v_{12} & =-\left|\begin{array}{ccc}
2 & \alpha_{2}-1 & \alpha_{1}+1 \\
\alpha_{2}+1 & 0 & \alpha+1 \\
\alpha-1 & \alpha+1 & 0
\end{array}\right|+y_{1}\left|\begin{array}{cc}
1+\alpha_{1} & \alpha_{1}+1 \\
\alpha_{2}+\alpha_{1} & \alpha+1
\end{array}\right| \\
& +y_{2}\left|\begin{array}{ll}
1+\alpha_{2} & \alpha_{2}+1 \\
\alpha_{2}+\alpha_{1} & \alpha+1
\end{array}\right|+y_{1} y_{2}\left(\alpha-\alpha_{1} \alpha_{2}\right)
\end{aligned}\right. \\
=\left\{2(\alpha+1)+y_{1} y_{2}\right\}\left(\alpha-\alpha_{1} \alpha_{2}\right)+\left\{\left(1+\alpha_{1}\right) y_{1}+\left(1+\alpha_{2}\right) y_{2}\right\}\left(\alpha+1-\alpha_{1}-\alpha_{2}\right) .
\end{aligned}
$$




\section{\$. 4.}

Diese Ausdrücke vereinfachen sich nun indem man sich, wie schon oben angedeutet, der elliptischen Funktionen bedient.

Setzt man zunächst

$$
\text { (21.) } \quad y_{i}=z_{i} \frac{\sqrt{2 \cdot \alpha+1 \cdot \alpha_{1}+1 \cdot \alpha_{2}+1}}{\alpha_{i}+1},
$$

so gehen die Gleichungen (20.) über in

wo

$$
\left\{\begin{array}{l}
v_{01}=2 \cdot \alpha-1 \cdot \alpha_{1}+1 \cdot \alpha_{2}+1 \cdot\left\{1+s^{2}+2 p z\right\} \\
v_{12}=2 \cdot \alpha+1 \cdot \alpha-\alpha_{1} \alpha_{2} \cdot\left\{1+z_{1} z_{2}+q_{0}\left(z_{1}+z_{2}\right)\right\}
\end{array}\right.
$$

(23.)

$$
p=\frac{\left(\alpha_{1}+\alpha_{2}+\alpha+1\right)}{\sqrt{2 \cdot \alpha+1 \cdot \alpha_{1}+1 \cdot \alpha_{2}+1}} \quad q_{0}=\frac{\alpha+1-\alpha_{1}-\alpha_{2}}{\alpha-\alpha_{1} \alpha_{2}} \cdot \sqrt{\frac{\alpha_{1}+1 \cdot \alpha_{2}+1}{2 \cdot \alpha+1}}
$$

Erinnert man sich jetzt der identischen Gleichung

$$
1+x y+\delta(x+y)=\frac{1+\delta}{2}(1+x)(1+y)+\frac{1-\delta}{2}(1-x)(1-y),
$$

so wird auch

$$
\left\{\begin{array}{l}
v_{00}=2 \cdot \alpha-1 \cdot \alpha_{1}+1 \cdot \alpha_{2}+1 \cdot\left\{\frac{1+p}{2}(1+z)^{2}+\frac{1-p}{2}(1-z)^{2}\right\} \\
v_{12}=2 \cdot \alpha+1 \cdot \alpha-\alpha_{1} \alpha_{2} \cdot\left\{\frac{1+q_{0}}{2}\left(1+z_{1}\right)\left(1+z_{2}\right)+\frac{1-q_{0}}{2}\left(1-z_{1}\right)\left(1-z_{2}\right)\right\}
\end{array}\right.
$$

Nun setze ich

$$
\text { (25.) } \quad \frac{1-z_{i}}{1+z_{i}}=\cos \text { am } v_{i} \quad \text { und } \quad k^{2}=\frac{1-p}{2} \quad k_{1}^{2}=\frac{1+p}{2} \text {. }
$$

Alsdann gehen die zwischen den $v$ bestehenden Gleichungen (16.) durch die Substitutionen

$$
\begin{aligned}
& v_{(x)}=2 . \alpha-1 . \alpha_{1}+1 . \alpha_{2}+1 .(1+z)^{2} \cdot \Delta^{2} \text { am } v \\
& v_{12}=2 . \alpha+1 . \alpha-\alpha_{1} \alpha_{2} .1+z_{1} .1+z_{2}\left\{\frac{1+\eta_{0}}{2}+\frac{1-\eta_{0}}{2} \cos \text { am } v_{1} \cos \text { am } v_{2}\right\}
\end{aligned}
$$

in die folgenden über:

$$
\text { (26.) }\left\{\begin{array}{l}
\Delta^{2} \text { am } v_{1} \Delta^{2} \text { am } v_{2}=\frac{\left(\alpha-\alpha_{1} \alpha_{2}\right)^{2}}{\alpha_{1}^{2}-1 . \alpha_{2}^{2}-1}\left\{\frac{1+q_{0}}{2}+\frac{1-q_{0}}{2} \cos \text { am } v_{1} \cos \text { am } v_{2}\right\}^{2} \\
\Delta^{2} \text { am } v_{2} A^{2} \text { am } v=\frac{\left(\alpha_{1}-\alpha_{2} \alpha\right)^{2}}{\alpha_{2}^{2}-1 \cdot \alpha^{2}-1}\left\{\frac{1+q_{1}}{2}+\frac{1-q_{1}}{2} \cos \text { am } v_{2} \cos \text { am } v\right\}^{2} \\
\Delta^{2} \text { am } v \Delta^{2} \text { am } v=\frac{\left(\alpha_{2}-\alpha \alpha_{1}\right)^{2}}{\alpha^{2}-1 \cdot \alpha_{1}^{2}-1}\left\{\frac{1+q_{2}}{2}+\frac{1-q_{2}}{2} \cos \text { am } v \cos \text { am } v_{1}\right\}^{2}
\end{array}\right.
$$


25. Clebsch, das Malfattische Problem auf den Oberflüchen $2^{\text {ter }}$ Ordnung. 301

(26.) $A^{2}$ am $v_{1} \Delta^{2}$ am $v_{2} A^{2}$ am $v$

$=\frac{\alpha-\alpha_{1} \alpha_{2} \cdot \alpha_{1}-\alpha_{2} \alpha \cdot \alpha_{2}-\alpha \alpha_{1}}{\alpha^{2}-1 \cdot \alpha_{1}^{2}-1 \cdot \alpha_{2}^{2}-1} \cdot\left\{\frac{1+q_{0}}{2}+\frac{1-q_{0}}{1} \cos \operatorname{am} v_{1} \cos\right.$ am $\left.v_{2}\right\}$

$\times\left\{\frac{1+q_{1}}{2}+\frac{1-q_{1}}{2} \cos\right.$ am $\left.v_{2} \cos \operatorname{am} v\right\}\left\{\frac{1+q_{2}}{2}+\frac{1-q_{2}}{2} \cos\right.$ am $v \cos$ am $\left.v_{1}\right\}$.

Die ersten drei dieser Gleichungen nun erinnern unwillkürlich an die Form des Additionstheorems der elliptischen Funktionen, welche unter Anderm sich bei Jacobi Bd. XXXIX p. 325 d. J. und Opusc. Math. II. p. 171 findet:

$\Delta \operatorname{am} u \Delta \operatorname{am} v \Delta \operatorname{am}(u+v)=k_{1}^{2}+k^{2} \cos$ am $u \cos$ am $v \cos$ am $\left.u+v\right)$.

Damit die obigen Gleichungen aber mit dieser vollständig übereinstimmen, ist es nöthig nachzuweisen, dafs man setzen dürfe:

$$
\left\{\begin{array}{l}
\frac{\left(\alpha-\alpha_{1} \alpha_{2}\right)}{\sqrt{\alpha_{1}^{2}-1 \cdot \alpha_{2}^{2}-1}} \cdot \frac{1+q_{0}}{2}=\frac{k_{1}^{2}}{\Delta \operatorname{am}(u+v)} \\
\frac{\alpha-\alpha_{1} \alpha_{2}}{\sqrt{\alpha_{1}^{2}-1 \cdot \alpha_{2}^{2}-1}} \cdot \frac{1-q_{0}}{2}=\frac{h^{2} \cos \operatorname{am}(u+v)}{\Delta \operatorname{am}(u+v)}
\end{array}\right.
$$

d. h. es mufs die identische Gleichung erfült sein, welche entsteht, wenn man diese beiden quadrirt, die erste mit $k^{2}=\frac{1-p}{2}$ multiplicirt, die zweite mit $k_{1}^{2}=\frac{1+p}{2}$, und sie dann addirt. So erhalten wir rechts $k^{2} k_{1}^{2}=\frac{1-p^{2}}{4}$, und

$$
\frac{1-p^{2}}{4}=\frac{\left(\alpha-\alpha_{1} \alpha_{2}\right)^{2}}{\alpha_{1}^{2}-1 \cdot \alpha_{2}^{2}-1}\left\{\left(\frac{1+q_{0}}{2}\right)^{2} \cdot \frac{1+p}{2}+\left(\frac{1-q_{0}}{2}\right)^{2} \cdot \frac{1-p}{2}\right\}
$$

Führt man rechts die Quadrate in der Parenthese aus, so kommt

oder

$$
1-p^{2}=\frac{\left(\alpha-\alpha_{1} \alpha_{2}\right)^{2}}{\alpha_{1}^{2}-1 \cdot \alpha_{2}^{2}-1}\left\{1+q_{0}^{2}+2 q_{0} p\right\}
$$

$$
\begin{gathered}
\alpha_{1}^{2}-1 \cdot \alpha_{2}^{2}-1\left\{1-\frac{\left(\alpha_{1}+\alpha_{2}+\alpha+1\right)^{2}}{2 \cdot \alpha_{1}+1 \cdot \alpha_{2}+1 \cdot \alpha+1}\right\} \\
=\left(\alpha-\alpha_{1} \alpha_{2}\right)^{2}\left\{1+\frac{\left(\alpha+1-\alpha_{1}-\alpha_{2}\right)^{2}}{\left(\alpha-\alpha_{1} \alpha_{2}\right)^{2}} \cdot \frac{\alpha_{1}+1 \cdot \alpha_{2}+1}{2 \cdot \alpha+1}+\frac{(\alpha+1)^{2}-\left(\alpha_{1}+\alpha_{2}\right)^{2}}{\alpha-\alpha_{1} \alpha_{2}} \cdot \frac{1}{\alpha+1}\right\} .
\end{gathered}
$$

Giebt man dieser Gleichung die Form

$$
\begin{gathered}
\alpha_{1}^{2}-1 . \alpha_{2}^{2}-1-\left(\alpha-\alpha_{1} \alpha_{2}\right)^{2} \\
=\frac{\left(\alpha_{1}+\alpha_{2}+\alpha+1\right)^{2}}{2 \cdot \alpha+1} \cdot \alpha_{1}-1 . \alpha_{2}-1+\frac{\left(\alpha+1-\alpha_{1}-\alpha_{2}\right)^{2}}{2 . \alpha+1} \cdot \alpha_{1}+1 . \alpha_{2}+1 \\
+\frac{(\alpha+1)^{2}-\left(\alpha_{1}+\alpha_{2}\right)^{2}}{\alpha+1}\left(\alpha-\alpha_{1} \alpha_{2}\right),
\end{gathered}
$$


so sieht man leicht, dafs auf beiden Seiten die Determinante

$$
\left|\begin{array}{ccc}
1 & \alpha_{2} & \alpha_{1} \\
\alpha_{2} & 1 & \alpha \\
\alpha_{1} & \alpha & 1
\end{array}\right|
$$

steht, dafs die obige Gleichung also wirklich eine identische ist, wie wir auch aus den im Anfange aufgestellten Prinzipien zu schliefsen berechtigt waren.

Setzen wir also, den Gleichungen (27.) entsprechend

$$
\frac{1-q_{0}}{1+q_{0}}=\cos a m \quad \frac{1-q_{1}}{1+q_{1}}=\cos \operatorname{am} \beta_{1} \quad \frac{1-q_{2}}{1+q_{2}}=\cos a m \beta_{2}, .
$$

so gehen die Gleichungen (26.) über in

$$
\left\{\begin{array}{l}
k_{1}^{2}+k^{2} \cos a m v_{1} \cos a m v_{2} \cos \mathrm{am} \beta=(-1)^{m} \Delta \operatorname{am} v_{1} \Delta \operatorname{am} v_{2} \Delta \operatorname{am} \beta \\
k_{1}^{2}+k^{2} \cos \operatorname{am} v_{2} \cos \operatorname{am} v \cos \operatorname{am} \beta_{1}=(-1)^{m_{1}} \Delta \operatorname{am} v_{2} \Delta \operatorname{am} v \Delta \operatorname{am} \beta_{1} \\
k_{1}^{2}+k^{2} \cos \operatorname{am} v \cos \operatorname{am} v_{1} \cos \operatorname{am} \beta_{2}=(-1)^{m_{2}} \Delta \operatorname{am} v \Delta \operatorname{am} v_{1} \Delta \operatorname{am} \beta_{2}
\end{array}\right.
$$

und die letzte Gleichung giebt

$$
m+m_{1}+m_{2}=\text { gerade. }
$$

Alsdann aber zeigt die Theorie der elliptischen Funktionen, dafs die Grölsen $v$ den Gleichungen genügen müssen:

$$
\left\{\begin{array}{l}
v_{1}+v_{2}=\eta \beta+2 m\left(K+i \boldsymbol{K}^{\prime}\right)+4 m^{\prime} \boldsymbol{K} \\
\boldsymbol{v}_{2}+\boldsymbol{v}=\eta_{1} \beta_{1}+2 m_{1}\left(\boldsymbol{K}+i \boldsymbol{K}^{\prime}\right)+4 m_{1}^{\prime} \boldsymbol{K} \\
\boldsymbol{v}+\boldsymbol{v}_{1}=\eta_{2} \beta_{2}+2 m_{2}\left(\boldsymbol{K}+i \boldsymbol{K}^{\prime}\right)+4 m_{2}^{\prime} \boldsymbol{K}
\end{array}\right.
$$

wo $K, K^{\prime}$ die gewöhnliche Bedeutung haben, die $\eta \pm 1$ bedeuten, und die $\boldsymbol{m}, \boldsymbol{m}^{\prime}$ ganze Zahlen sind. Lösen wir die Gleichungen nach den $v$ auf, und lassen die geraden Vielfachen von $K+i K^{\prime}$ und $2 K$ in den Werthen der $v$ aus, da wir nur die cos am kennen wollen und diese hiedurch keine Veränderung erfahren, so erhalten wir

$$
\text { (31.) }\left\{\begin{array}{l}
v=\frac{-\eta \beta+\eta_{1} \beta_{1}+\eta_{2} \beta_{2}}{2}+2 m \boldsymbol{K} \\
v_{1}=\frac{\eta \beta-\eta_{1} \beta_{1}+\eta_{2} \beta_{2}}{2}+2 m \boldsymbol{K} \\
v_{2}=\frac{\eta \beta+\eta_{1} \beta_{1}-\eta_{2} \beta_{2}}{2}+2 m \boldsymbol{K}
\end{array}\right.
$$

wo $m$ eine ganze Zahl bedeutet. Da übrigens das Zeichen der $v$ beliebig ist, und keinen Einflufs auf $\cos$ am $v$ ausübt, so können wir eins der $\eta$ willkürlich bestimmen, und haben so nur 8 wesentlich verschiedene Systeme der $v$. 
\$. 5.

Es bleibt nur übrig noch die $b$ selbst zu bestimmen, nachdem wir durch die Gleichungen (21. 28. 31.) die $y$ bestimmt haben. Setzen wir die Determinante

$$
\left|\begin{array}{cccc}
a_{1} & a_{2} & a_{3} & a_{4} \\
a_{1}^{\prime} & a_{2}^{\prime} & a_{3}^{\prime} & a_{4}^{\prime} \\
a_{1}^{\prime \prime} & a_{2}^{\prime \prime} & a_{3}^{\prime \prime} & a_{4}^{\prime \prime} \\
b_{1} & b_{2} & b_{3} & b_{4}
\end{array}\right|=A_{1} b_{1}+A_{2} b_{2}+A_{3} b_{3}+A_{4} b_{4}
$$

so daf's die $\boldsymbol{A}$ die Koordinaten des Schnittpunkts der drei gegebenen Ebenen bedeuten; so folgt aus der Gleichung, welche den Beginn des $\$$. 3 bildete

$$
\begin{aligned}
\boldsymbol{P R}^{2} & =v_{(0)} \mu^{2}+v_{11} \mu^{\prime 2}+v_{22} \mu^{\prime \prime 2}+2 v_{01} u \mu^{\prime}+2 v_{12} \mu^{\prime} \mu^{\prime \prime}+2 v_{20} \mu^{\prime \prime} \mu \\
& =\Delta^{3} \cdot\left|\begin{array}{llll}
a_{1} & a_{1}^{\prime} & a_{1}^{\prime \prime} & b_{1} \lambda+b_{1}^{\prime} \lambda^{\prime}+b_{1}^{\prime \prime} \lambda^{\prime \prime} \\
a_{2} & a_{2}^{\prime} & a_{2}^{\prime \prime} & b_{2} \lambda+b_{2}^{\prime} \lambda^{\prime}+b_{2}^{\prime \prime} \lambda^{\prime \prime} \\
a_{3} & a_{3}^{\prime} & a_{3}^{\prime \prime} & b_{3} \lambda+b_{3}^{\prime} \lambda^{\prime}+b_{3}^{\prime \prime} \lambda^{\prime \prime} \\
a_{4} & a_{4}^{\prime} & a_{4}^{\prime \prime} & b_{4} \lambda+b_{4}^{\prime} \lambda^{\prime}+b_{4}^{\prime \prime} \lambda^{\prime \prime}
\end{array}\right|,
\end{aligned}
$$

indem wir aul beiden Seiten die Coefficienten der $\lambda$ vergleichen:

$$
\left\{\begin{array}{c}
\left\{A_{1} b_{1}+A_{2} b_{2}+A_{3} b_{3}+A_{4} b_{4}\right\}^{2}=\frac{v_{00}}{J^{3}} \\
\left\{A_{1} b_{1}^{\prime}+A_{2} b_{2}^{\prime}+A_{3} b_{3}^{\prime}+A_{4} b_{4}^{\prime}\right\}\left\{A_{1} b_{1}^{\prime \prime}+A_{2} b_{2}^{\prime \prime}+A_{3} b_{3}^{\prime \prime}+A_{4} b_{4}^{\prime \prime}\right\}=\frac{\varepsilon_{1}^{0} \varepsilon_{2}^{0} v_{12}}{J^{3}} .
\end{array}\right.
$$

Die erste dieser Gleichungen giebt

$$
\text { (34.) } \quad A_{1} b_{1}+A_{2} b_{2}+A_{3} b_{3}+A_{4} b_{4}=\zeta_{0} \sqrt{\frac{v_{00}}{\Delta^{3}}}
$$

wo $\zeta= \pm 1$. Da aber alsdann die zweite ergiebt

$$
\text { (34 a.) } \quad \zeta_{1} \zeta_{2} \sqrt{v_{11} v_{22}}=\varepsilon_{1}^{0} \varepsilon_{2}^{0} v_{12} \text {, }
$$

so ist, wenn wir den Gröfsen $\sqrt{v_{(x)}}$ etc. eine feste Bedeutung beilegen von den $\zeta$ nur ein einziges willkürlich, so dafs es also nur zwei Systeme der $\zeta$ giebt. Man sieht, dafs an dieser Stelle zum letzten Male eine Zweideutigkeit entsteht, da von hier ab nur mit linearen Gleichungen zu operiren ist. Daher können wir schon die Anzahl der möglichen Lösungen angeben, welche auf 64 steigt.

Die Gleichung (34.) verbinden wir nun mit den Gleichungen

$$
\mid \begin{aligned}
& \Sigma \Sigma \Delta_{i k} b_{i} a_{k}=-\varepsilon_{0}^{\prime \prime}\left(1+y_{0}\right) \\
& \Sigma \Sigma \Delta_{i k} b_{i} a_{k}^{\prime}=-\varepsilon_{0}^{\prime} \\
& \Sigma \Sigma \Delta_{i k} b_{i} a_{k}^{\prime \prime}=-\varepsilon_{0}^{\prime \prime}
\end{aligned}
$$

Journal f. d. M. Bd. LIII. Heft 4. 
304 23. Clebsch, das Malfattische Problem auf den Oberfä̈chen $2^{\text {ter }}$ Ordnung.

So haben wir 4 lineare Gleichungen zur Bestimmung von $b_{1}, b_{2}, b_{3}, b_{4}$. Setzt man nun für den Augenblick

$$
\text { (36.) } \quad \omega_{i}=\Delta_{i 1} b_{1}+\Delta_{i 2} b_{2}+\Delta_{i 3} b_{3}+\Delta_{i 4} b_{4}
$$

und bezeichnet mit $\boldsymbol{U}$ den Ausdruck in welchen die Funktion $u$ für die Koordinaten des Schnittpunkts der gegebenen Ebenen übergeht

$$
\text { (37.) } U=\Sigma \Sigma u_{i k} A_{i} A_{k} \text {, }
$$

so gehen die Gleichungen (34.35.) in diese über:

$$
\left\{\begin{array}{c}
\frac{1}{2}\left\{\omega_{1} \frac{\partial U}{\partial A_{1}}+\omega_{2} \frac{\partial U}{\partial A_{2}}+\omega_{3} \frac{\partial U}{\partial A_{3}}+\omega_{4} \frac{\partial U}{\partial A_{4}}\right\}=\zeta_{0} \sqrt{\frac{v_{00}}{\Delta}} \\
\omega_{1} a_{1}+\omega_{2} a_{2}+\omega_{3} a_{3}+\omega_{4} a_{4}=-\varepsilon_{0}^{\prime}\left(1+y_{01}\right) \\
\omega_{1} a_{1}^{\prime}+\omega_{2} a_{2}^{\prime}+\omega_{3} a_{3}^{\prime}+\omega_{4} a_{4}^{\prime}=-\varepsilon_{0}^{\prime} \\
\omega_{1} a_{1}^{\prime \prime}+\omega_{2} a_{2}^{\prime \prime}+\omega_{3} a_{3}^{\prime \prime}+\omega_{4} a_{4}^{\prime \prime}=-\varepsilon_{0}^{\prime \prime} .
\end{array}\right.
$$

Die Determinante dieses Systems ist $\boldsymbol{U}$; ihr Differentialquotient nach dem Elemente $\frac{1}{2} \frac{\partial U}{\partial A_{i}}$ ist $\boldsymbol{A}_{i}$, und ihr Differentialquotient nach $\boldsymbol{a}_{i}^{k}$ ist der vollständige Differentialquotient $\frac{1}{2} \frac{\partial U}{\partial a_{i}^{k}}$. Die Auflösung der Gleichungen giebt also:

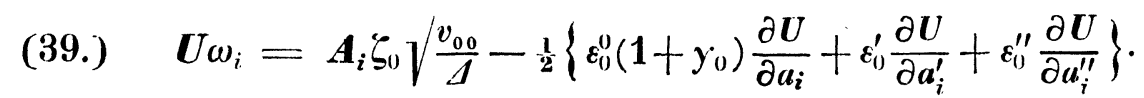

Die Gleichung der Ebene $\boldsymbol{B}$ läfst sich in der Form darstellen:

$$
2 \Delta \boldsymbol{B}=\omega_{1} \frac{\partial u}{\partial x_{1}}+\omega_{2} \frac{\partial u}{\partial x_{2}}+\omega_{3} \frac{\partial u}{\partial x_{3}}+\omega_{4} \frac{\partial{ }^{\prime 6}}{\partial x_{4}} .
$$

Führt man hier die Werthe der $\omega$ ein, und setzt

so wird

$$
\begin{gathered}
\boldsymbol{X}=\frac{1}{2 \sqrt{-\Delta}}\left\{\boldsymbol{A}_{1} \frac{\partial u}{\partial x_{1}}+\boldsymbol{A}_{2} \frac{\partial u}{\partial x_{2}}+\boldsymbol{A}_{3} \frac{\partial u}{\partial x_{3}}+\boldsymbol{A}_{4} \frac{\partial u}{\partial x_{4}}\right\} \\
\boldsymbol{X}^{(k)}=\frac{1}{2} \sum_{i=1}^{i=4} \frac{\partial U}{\partial a_{i}^{k}} \cdot \frac{\partial u}{\partial x_{i}},
\end{gathered}
$$

$$
\text { A. U. } \boldsymbol{B}=\zeta_{0} \sqrt{-v_{(x)}} \cdot \boldsymbol{X}-\left\{\varepsilon_{0}^{0}\left(1+y_{0}\right) \boldsymbol{X}^{0}+\varepsilon_{10}^{\prime} \boldsymbol{X}^{\prime}+\varepsilon_{0}^{\prime \prime} \boldsymbol{X}^{\prime \prime}\right\}
$$

oder indem man mit $\varepsilon_{0}^{0}$ multiplicirt:

$$
\begin{gathered}
\varepsilon_{10}^{\prime \prime} \Delta \boldsymbol{U} \boldsymbol{B}=\zeta_{0} \varepsilon_{10}^{\prime \prime} \sqrt{-v_{00}} \boldsymbol{X}+\left\{-\left(1+y_{0}\right) \boldsymbol{X}^{\prime \prime}+\varepsilon_{2}^{\prime} \varepsilon_{2}^{\prime \prime} \boldsymbol{X}^{\prime}+\varepsilon_{1}^{\prime \prime} \varepsilon_{1}^{\prime \prime} \boldsymbol{X}^{\prime \prime}\right\} \\
\sqrt{-v_{(0)}}=\sqrt{2.1-\alpha \cdot \alpha_{1}+1 \cdot \alpha_{2}+1} \cdot \frac{2 \Delta \operatorname{am} v_{0}}{1+\cos a m v_{0}} \\
y_{01}=\sqrt{\frac{2 \cdot \alpha_{1}+1 \cdot \alpha_{2}+1}{\alpha+1}} \cdot \frac{1-\cos \mathrm{am} v_{0}}{1+\cos \mathrm{am} v_{0}} .
\end{gathered}
$$


In Betreff der $\zeta$ ist noch eine Bemerkung zu machen. Bei der fûr $\sqrt{-v_{(0)}}$ gewählten Bedeutung giebt die Gleichung (34 a.)

Also

$$
\zeta^{\prime \prime} \zeta^{\prime}=\varepsilon_{10}^{\prime \prime} \varepsilon_{1}^{\prime \prime} \quad \zeta^{\prime} \zeta^{\prime \prime}=\varepsilon_{1}^{\prime 1} \varepsilon_{2}^{0} \quad \zeta^{\prime \prime} \zeta^{\prime \prime}=\varepsilon_{0,}^{\prime} \varepsilon_{11}^{\prime \prime} .
$$

$$
\zeta^{(1)} \varepsilon_{0}^{(\prime)}: \zeta^{\prime \prime} \varepsilon_{1}^{\prime}: \zeta^{\prime \prime} \varepsilon_{2}^{\prime \prime}=\varepsilon_{10}^{\prime} \varepsilon_{0}^{\prime \prime}: \varepsilon_{1}^{\prime \prime} \varepsilon_{1}^{\prime \prime}: \varepsilon_{2}^{\prime \prime} \varepsilon_{2}^{\prime} ;
$$

so dafs die Gleichungen der sämmtlichen gesuchten Ebenen folgende sind:

$$
\left\{\begin{array}{l}
0=\zeta . \varepsilon_{0}^{\prime} \varepsilon_{01}^{\prime \prime} \sqrt{-v_{00}} \boldsymbol{X}+\left\{-\left(1+y_{0}\right) \boldsymbol{X}^{0}+\varepsilon_{2}^{\prime} \varepsilon_{2}^{0} \boldsymbol{X}^{\prime}+\varepsilon_{1}^{0} \varepsilon_{1}^{\prime \prime} \boldsymbol{X}^{\prime \prime}\right\} \\
0=\zeta . \varepsilon_{1}^{\prime \prime} \varepsilon_{1}^{0} \sqrt{-v_{11}} \boldsymbol{X}+\left\{\varepsilon_{2}^{\prime} \varepsilon_{2}^{0} \boldsymbol{X}^{0}-\left(1+y_{1}\right) \boldsymbol{X}^{\prime}+\varepsilon_{11}^{\prime \prime} \varepsilon_{1}^{\prime} \boldsymbol{X}^{\prime \prime}\right\} \\
0=\zeta . \varepsilon_{2}^{0} \varepsilon_{2}^{\prime} \sqrt{-v_{22}} \boldsymbol{X}+\left\{\varepsilon_{1}^{0} \varepsilon_{1}^{\prime \prime} \boldsymbol{X}^{0}+\varepsilon_{0}^{\prime} \varepsilon_{01}^{\prime \prime} \boldsymbol{X}^{\prime}-\left(1+y_{2}\right) \boldsymbol{X}^{\prime \prime}\right\} \quad \zeta= \pm 1
\end{array}\right.
$$

Man sieht, dafs von den $\varepsilon$ nur die Verbindungen $\varepsilon_{0}^{\prime} \varepsilon_{1}^{\prime \prime}, \varepsilon_{1}^{0} \varepsilon_{1}^{\prime \prime}, \varepsilon_{2}^{\prime \prime} \varepsilon_{2}^{\prime}$ eingehen, welche auch in den $y$ allein vorkommen. Dies würde also vier Gruppen von Lösungen gebell, die verschiedenen Werlhe der $\cos$ am $v$ acht, und die $\zeta$ zwei, so dafs man 64 Lösungen erhält.

\section{\$. 6.}

Herr Prof. Schellbach hat eine Auflösung des vorliegenden Problems für den Fall einer Kugel bekannt gemacht, welche von drei Ebenen in gröfsten Kreisen geschnitten wird (45ster Band dieses Journals) und die dort gegebenen Formeln sind zunächst die Veranlassung zu der vorliegenden Untersuchung gewesen. Jene Formeln indefs lassen sich unmittelbar auf die allgemeine Aufgabe ausdehnen, bei welcher statt der Ebenen dreier gröfsten Kreise irgend andere gegeben sind. Sei die Gleichung der Kugel

$$
u=x_{1}^{2}+x_{2}^{2}+x_{3}^{2}-x_{4}^{2}=0
$$

und die Gleichungen der gegebenen und gesuchten Kreise

$$
\begin{aligned}
A^{i} \sin \vartheta^{i} & =x_{1} \cos a_{1}^{i}+x_{2} \cos a_{2}^{i}+x_{3} \cos a_{3}^{i}-x_{4} \cos \vartheta^{i}=0 \\
\varepsilon_{i}^{i} \boldsymbol{B}^{i} \sin \theta^{i} & =x_{1} \cos b_{1}^{i}+x_{2} \cos b_{2}^{i}+x_{3} \cos b_{3}^{i}-x_{4} \cos \theta^{i}=0 .
\end{aligned}
$$

Dann wird

$$
\text { (40 a.) }\left\{\begin{array}{c}
\alpha_{i}=\frac{\cos a_{1}^{h} \cos a_{1}^{k}+\cos a_{2}^{h} \cos a_{2}^{k}+\cos a_{3}^{h} \cos a_{3}^{k}-\cos \vartheta^{h} \cos \vartheta^{k}}{\sin \vartheta^{h} \sin \vartheta^{k}} \\
=\frac{\cos V^{i}-\cos \vartheta^{h} \cos \vartheta^{k}}{\sin \vartheta^{h} \sin \vartheta^{k}}=\cos \delta^{i}
\end{array}\right.
$$

wenn $V^{i}$ die Seiten des von den gegebenen Kreismittelpankten gebildeten sphärischen Dreiecks bedeuten, $\delta^{i}$ die Winkel, welche die von zwei Ecken dieses Dreiecks nach den Schnittpunkten ihrer Kreise gezogenen sphärischen Bogen mit einander bilden. 
Nun denke man sich ein sphärisches Dreieck construirt, dessen Winkel $I^{\prime}, I_{1}, I_{2}^{\prime}$ und dessen Seiten $g, g_{1}, g_{2}$, welches dadurch bestimmt ist, dafs (40 b.) $\cos T=-\varepsilon_{0}^{\prime} \varepsilon_{0}^{\prime \prime} \cos \delta^{\prime \prime}$ etc.

Die vier verschiedenen Dreiecke, welche den verschiedenen Systemen der $\varepsilon$ entsprechen, werden durch dieselben gröfsten Kreise gebildet, und wenn man durch $T, T_{1}, I_{2}^{\prime}, g, g_{1}, g_{2}$ Winkel und Seiten eines derselben bezeichnet, so sind die der übrigen:

$$
\begin{array}{rrrrrr}
I^{\prime} & \pi-\Gamma_{1} & \pi-I_{2} & g & \pi-g_{1} & \pi-g_{2} \\
\pi-I^{\prime} & \Gamma_{1} & \pi-I_{2} & \pi-g & g_{1} & \pi-g_{2} \\
\pi-I^{\prime} & \pi-\Gamma_{1} & \Gamma_{2} & \pi-g & \pi-g_{1} & g_{2} .
\end{array}
$$

Man hat sodann aus (27. 28.), nach bekannten Formeln:

und

$$
\left\{\begin{array}{c}
\cos \operatorname{am} \beta_{i}=\frac{\sin \frac{\Gamma_{h}}{2} \sin \frac{\Gamma_{k}}{2}-\sin \frac{\Gamma_{i}}{2}}{\sin \frac{\Gamma_{h}}{2} \sin \frac{\Gamma_{k}}{2}+\sin \frac{\Gamma_{i}}{2}}=\operatorname{ctg} \frac{s}{2} \operatorname{tg}\left(\frac{s}{2}-g_{i}\right) \\
\Delta \operatorname{am} \beta_{i}=\frac{\cos \frac{\Gamma_{h}}{2} \cos \frac{\Gamma_{k}}{2}}{\sin \frac{\Gamma_{h}}{2} \sin \frac{\Gamma_{k}}{2}+\sin \frac{\Gamma_{i}}{2}}=\frac{\cos \frac{s}{2}}{\cos \left(\frac{s}{2}-g_{i}\right)} \\
2 s=g_{1}+g_{2}+g
\end{array}\right.
$$

$$
k^{2}=\sin ^{2} \frac{s}{2} \quad k_{1}^{2}=\cos ^{2} \frac{s}{2},
$$

so dafs der Modul durch den Umfang des construirten sphärischen Dreiecks unmittelbar gegeben ist.

Man kann sich nun durch die Analogie bestimmen lassen, drei Winkel $\alpha, \alpha_{1}, \alpha_{2}$ zu suchen, welche die Bedingungen erfüllen:

$$
\text { (42.) }\left\{\begin{array}{l}
\cos \operatorname{am}\left(\beta_{h}+\beta_{k}\right)=\operatorname{ctg} \frac{s}{2} \operatorname{tg} \alpha_{i} \\
\Delta \operatorname{am}\left(\beta_{h}+\beta_{k}\right)=\frac{\cos \frac{s}{2}}{\cos \alpha_{i}} .
\end{array}\right.
$$

Dann sieht man aus dem oben angeführten Additionstheorem, dafs die $\alpha$ den Gleichungen genügen :

$$
1=\frac{\cos \alpha_{i} \cos \left(\frac{s}{2}-g_{h}\right) \cos \left(\frac{s}{2}-g_{k}\right)}{\cos \frac{s}{2}}-\frac{\sin \alpha_{i} \sin \left(\frac{s}{2}-g_{h}\right) \sin \left(\frac{s}{2}-g_{k}\right)}{\sin \frac{s}{2}}
$$


25. Clebsch, das Malfattische Problem auf den Oberfü̈chen $2^{\text {ter }}$ Ordnung. 307 und diejenigen Wurzeln dieser Gleichungen, welche nicht die Gleichungen (43.) erfüllen, genügen den folgenden:

$$
\text { (44.) }\left\{\begin{array}{l}
\cos \operatorname{am}\left(\beta_{h}-\beta_{k}\right)=\operatorname{ctg} \frac{s}{2} \operatorname{tg} \alpha_{i}^{\prime} \\
\Delta \operatorname{am}\left(\beta_{k}-\beta_{k}\right)=\frac{\cos \frac{s}{2}}{\cos \alpha_{i}^{\prime}} .
\end{array}\right.
$$

Bemerken wir nun die identischen Gleichungen

$$
\begin{aligned}
\cos ^{2} \mathrm{am} \frac{u}{2} & =\frac{k_{1}^{2}}{k^{2}} \cdot \frac{1-\Delta \mathrm{am} u}{\Delta \mathrm{am} u-\cos \mathrm{am} u} \\
\Delta^{2} \mathrm{am} \frac{u}{2} & =k_{1}^{2} \cdot \frac{1-\cos \mathrm{am} u}{\Delta \mathrm{am} u-\cos \mathrm{am} u},
\end{aligned}
$$

welche man unter Anderm aus dem erwähnten Additionstheorem ohne Weiteres findet, so haben wir aus (41. 42. etc.)

$$
\begin{aligned}
\hat{\beta}^{2} \mathrm{am} \frac{\beta_{i}}{2} & =\cos \frac{s}{2} \frac{\cos \frac{s-g_{i}}{2}}{\cos \frac{g_{i}}{2}} \\
\cos ^{2} \mathrm{am} \frac{\beta_{i}}{2} & =\operatorname{ctg} \frac{s}{2} \operatorname{tg} \frac{g_{i}}{2} \\
\sin ^{2} \mathrm{am} \frac{\beta_{i}}{2} & =\frac{\sin \frac{s-g_{i}}{2}}{\sin \frac{s}{2} \cos \frac{g_{i}}{2}}
\end{aligned}
$$

und eben dieselben Formeln für $\frac{\beta_{h}+\beta_{k}}{2}$ und $\frac{\beta_{h}-\beta_{k}}{2}$, wo denn $\frac{s}{2}-\alpha_{i}$ und $\frac{s}{2}-\alpha_{i}^{\prime}$ an die Stelle von $g_{i}$ tritt.

Und indem man nun die Gleichungen Fundamenta p. 33 (10-12.) anwendet, wird

$$
\begin{aligned}
& \Delta \mathrm{am} \frac{\beta_{i}+\beta_{h}+\beta_{k}}{2} \Delta \mathrm{am} \frac{-\beta_{i}+\beta_{h}+\beta_{k}}{2}=\cos \frac{s}{2} \frac{\cos \frac{g_{i}+\alpha_{i}-\frac{s}{2}}{2}}{\cos \frac{g_{i}-\alpha_{i}+\frac{s}{2}}{2}} \\
& \cos \mathrm{am} \frac{\beta_{i}+\beta_{h}+\beta_{k}}{2} \cos \mathrm{am} \frac{-\beta_{i}+\beta_{h}+\beta_{k}}{2}=\operatorname{ctg} \frac{s}{2} \operatorname{tg} \frac{y_{i}-\alpha_{i}-\frac{s}{2}}{2}
\end{aligned}
$$


308 25. Clebsch, das Malfattische Problem auf den Oberflüchen $2^{\text {ter }}$ Ordnung.

$$
\begin{aligned}
& \sin \mathrm{am} \frac{\beta_{i}+\beta_{h}+\beta_{k}}{2} \cos \mathrm{am} \frac{-\beta_{i}+\beta_{h}+\beta_{k}}{2}=\frac{\sin \frac{g_{i}+\alpha_{i}-\frac{s}{2}}{2}}{\sin \frac{s}{2} \cos \frac{g_{i}-\alpha_{i}^{\prime}-\frac{s}{2}}{2}} \\
& \Delta \mathrm{am} \frac{\beta_{i}+\beta_{h}-\beta_{k}}{2} \Delta \operatorname{am} \frac{\beta_{i}-\beta_{h}+\beta_{k}}{2}=\cos \frac{s}{2} \frac{\cos \frac{y_{i}+\alpha_{i}^{\prime}-\frac{s}{2}}{2}}{\cos \frac{y_{i}-\alpha_{i}^{\prime}-\frac{s}{2}}{2}} \\
& \cos \operatorname{am} \frac{\beta_{i}+\beta_{h}-\beta_{k}}{2} \cos \operatorname{am} \frac{\beta_{i}-\beta_{h}+\beta_{k}}{2}=\operatorname{ctg} \frac{s}{2} \operatorname{tg} \frac{g-\alpha_{i}^{\prime}-\frac{s}{2}}{2} \\
& \sin \operatorname{am} \frac{\beta_{i}+\beta_{h}-\beta_{k}}{2} \cos \mathrm{am} \frac{\beta_{i}-\beta_{h}+\beta_{k}}{2}=-\frac{\sin \frac{\dot{g_{i}+\alpha_{i}^{\prime}-\frac{s}{2}}}{2}}{\sin \frac{s}{2} \cos \frac{g_{i}-\alpha_{i}^{\prime}-\frac{s}{2}}{2}} .
\end{aligned}
$$

Diese Formeln, zusammen mit (43.) bilden die Verallgemeinerung der von Herrn Prof. Schellbach a. a. O. gegebenen, und sind genau mit denselben identisch. Auch sind dieselben natürlich für den oben ganz allgemein behandelten Fall gültig, wenn nur die $I^{\prime} g$ aus den Gleichungen $(40 a$. 40 b.) bestimmt werden; und sie dienen so gewissermafsen zur Umgehung der elliptischen Funktionen.

Berlin den 9. Dezember 1855. 